\title{
Special issue on formal diachronic semantics Introduction
}

\author{
IGOR YANOVICH \\ University of Tübingen \\ igor.yanovich@uni-tuebingen.de
}

It is a pleasure to present this collection of articles which make up the first-ever special issue dedicated specifically to formal diachronic semantics. This young subfield is in its formative stages, and is concerned with using the apparatus of "formal", that is, logical semantics, for understanding meaning change, particularly of grammatical meaning.

Formal diachronic semantics is not strictly delineated from other types of historical semantics, just as modern formal semantics is informed by the insights in usageconscious, cognitively-oriented, or sociolinguistically-aware research. Choosing the logical approach to linguistic meaning is not so much a matter of dogma or ideology as it is one of convenience: as it turns out, the logical perspective can often lead to interesting discoveries about how language functions which often complement those made by pursuing other lines of linguistic inquiry.

There have arguably been two key moments in the gradual emergence of formal diachronic semantics as a research field. The first one was the publication of Eckardt (2006), a book which, in hindsight, can be said to have created formal diachronic semantics as a field. Eckardt addressed both formal semanticists and historical linguists and tried, with several case studies, to show how starting from a logical analysis of meaning can contribute to a better understanding of the diachronic semantic processes.

An important insight of Eckardt's concerned the nature of a minimal change, and was addressed more to the formal semanticists in her audience: she noted that when speakers and hearers conduct semantic reanalysis - potentially leading to a lasting meaning change - the primary objects they operate on are sentence meanings, not those of individual lexical items or morphemes. A reanalysis by the hearer (from the speaker's perspective, a misanalysis of her words) must result in a different yet reasonable meaning for the whole utterance. From that global meaning, the hearer can then work out the meanings of the sentence's parts, which may involve assigning innovative lexical/morphemic meanings to some of them. When we write down the innovative meanings for words and morphemes using logical formalisms, they might look quite different from the original formulas describing the speaker's intended 
meaning. The insight here is that this change happens through the interaction between the speaker and hearer, at the discourse level, and not at the level of specific subformulas. The level of subformulas simply follows from the change that happened elsewhere; subformulas themselves are used as a tool for us to understand the relevant meanings, but not as objects directly manipulated by speakers and hearers in meaning change.

Thus, when we try to understand a meaning change, we should look for the actual contexts of reanalysis, rather than try to manipulate the symbols of our logical representations. This realization may go against the instinct of a semanticist used to working with formulas for individual lexical items and how they compose with each other; nevertheless, it is completely at home in a more holistic, and no less formal-semantic, tradition of studying both semantics and pragmatics with mathematical and philosophical tools. Moreover, Eckardt's insight also brings formal diachronic semantics immediately into close dialogue with the practices used in historical linguistics, where working with specific reanalysis contexts is a long-established practice.

The second foundational moment for the field was Ashwini Deo's organization of the workshop "Systematic Semantic Change" at the University of Texas at Austin in April 2013. This workshop brought together formal semanticists with historical linguists and typologists like Östen Dahl and Elizabeth Traugott. It was so successful that it sparked a series of subsequent events. First, a special session was organized at "Sinn und Bedeutung 19" in 2014 at the University of Göttingen by Regine Eckardt, Igor Yanovich, and Hedde Zeijlstra, with Hedde the main driving force behind it; and then in 2015, at the "22nd International Conference on Historical Linguistics" held at the University of Naples, another special session was organized by Cleo Condoravdi and Ashwini Deo. Subsequently, Regine Eckardt, recognizing that there was sufficient interest in this new subfield to warrant a regular conference series, organized the inaugural "Formal Diachronic Semantics" conference (FoDS) in 2016 at the University of Konstanz. Since then, FoDS has been held in 2017 at the University of Saarland (organized by Remus Gergel, Martin Kopf-Giammanco, Jonathan Watkins and Sylvia Monzon), in 2018 at the University of Oslo (organized by Patrick Grosz and Dag Haug); and in 2019 at the Ohio State University (organized by Ashwini Deo, Micha Elsner, Brian Joseph, Marie-Catherine de Marneffe and Andrea Sims). FoDS 5 was to be held in Jerusalem in the Spring of 2020, organized by Elitzur Bar-Asher Siegal, Nora Boneh, Eitan Grossman and Aynat Rubinstein, but has had to be postponed to the Fall of 2020 due to the SARS-CoV-2 pandemic.

Consequently, formal diachronic semantics presentations and discussions have become common at workshops. In principle, there would also exist by now a number of journal articles emerging from this new area of research, and there are certainly a few, such as Beck and Gergel (2015) or Deo (2015); yet most of the presentations at the above-mentioned formal diachronic semantics workshops have not led to such publications. For someone who took part in the abstract selection committees and who was present at the workshops themselves, this is not too surprising: while there is no shortage of very interesting puzzles in meaning change, there is a relative 
lack of successful methods available to help us make enough progress resolving those puzzles to warrant a journal publication.

This is where the (admittedly presumptuous) idea for the present special issue stemmed from. We wanted to help the subfield to hone its research strategies: partly by inviting more researchers in the field to express them in the format of an article, and partly by forcing the submitted papers to go through rigorous review. After all, it is an open secret that journal-published linguistic research often benefits tremendously from the generously given suggestions, questions, and ideas of the reviewers. We think that this has been the case for all of the papers submitted to the current special issue: those that appear in this volume, those still in the last stages of review for a subsequent issue, and even those that were declined.

This collection would not have been possible without the numerous anonymous reviewers, coming from different subfields of linguistics: formal semanticists, typologists, historical linguists, syntacticians... Because the papers submitted for the collection usually spanned several areas of expertise, up to four reviewers were called upon in a single round of reviewing, in order to provide a fair and rigorous assessment of all the relevant aspects of each paper. We are grateful beyond words to all those who gave to us their time, their care, their knowledge, and their wit in providing reviews for the articles in this special issue. Thank you very, very much!

The special issue would also not have been possible without the intellectual support and work of the editors-in-chief supervising the special issue: Elizabeth Cowper and Heather Newell. It is our great pleasure to thank them sincerely for all they did to help this issue be published!

Our hope is that when you read the four articles printed in this collection, you will note on the one hand how the authors use novel methodological approaches in their striving for rigour, precision, and truth; while noting on the other hand that there remain an enormous number of open questions for formal diachronic semantics. Some of these are only beginning to emerge and for many there are as yet no established and proven research methods. There is a lot of work still to be done. We hope you will enjoy the serious attempts made in the articles in this issue to bring us all to a better understanding of how meanings change over time, one step at a time.

Igor Yanovich (on behalf of myself, Regine Eckardt and Dag Haug)

\section{REFERENCES}

Beck, Sigrid and Remus Gergel. 2015. The diachronic semantics of English again. Natural Language Semantics 23(3): 157-203.

Deo, Ashwini. 2015. The semantic and pragmatic underpinnings of grammaticalization paths: The progressive to imperfective shift. Semantics and Pragmatics 8(14): 1-52.

Eckardt, Regine. 2006. Meaning change in grammaticalization. An enquiry into semantic reanalysis. Oxford: Oxford University Press. 